\title{
Novel risk factors for coronary artery disease - role of testosterone and high-sensitivity CRP in Sri Lankan males
}

\author{
Wickramatilake $\mathbf{C} \mathbf{M}^{1}$ \\ Journal of the Ceylon College of Physicians, 2016, 47, 70-75
}

DOI: http://doi.org/10.4038/jccp.v47i2.7786

\begin{abstract}
Coronary artery disease (CAD) is multi-factorial in origin and develops as a result of both genetic and environmental factors. Traditional risk factors (generally smoking, hypertension, raised lipids, and diabetes mellitus) do not explain all of the risk for coronary disease events. There remain several other factors that contribute to the overall risk. Various new or emerging risk factors have been studied to improve global risk assessment for CAD. Inflammation indicated by markers as Creactive protein and low androgen (testosterone) status in males are among the emerging risk factors. In the local setting, the association of testosterone with CAD has not been explored, although this relationship has been well-studied in the Western population. Therefore we investigated, whether serum levels of total testosterone (TT) and high sensitivity C-reactive protein (hs-CRP) differ in men with CAD from those without CAD and to evaluate the relationship of serum TT and hs-CRP with the severity of CAD.

Three hundred and nine males (103 patients with ST-elevation myocardial infarction (STEMI), 103 patients with angiographically-proven CAD, 103 controls without having a history of CAD) were studied. Serum TT, hs-CRP, lipids, cardiac troponin I (cTnl) and plasma glucose were estimated. Three angiogram-based severity scores were used in the severity assessment of angiographically-proven CAD. In STEMI patients, clinical risk scores and modified Selvester ECG QRS score were used in assessing the severity of myocardial infarction.
\end{abstract}

\footnotetext{
${ }^{1}$ Department of Biochemistry, Faculty of Medicine, University of Ruhuna, Sri Lanka.
}

Correspondence: CMW

E-mail: chandimadhu@live.com
We showed that low levels of total testosterone, high levels of hs-CRP, abnormal lipid profile and raised plasma glucose contribute to an atherogenic milieu. Low levels of total testosterone and high level of hs-CRP can be perceived as risk factors of coronary artery disease.

\section{Introduction}

Coronary artery disease (CAD) is a condition in which atherosclerotic plaque builds up within the wall of the coronary arteries leading to their narrowing and the clinical manifestations of acute coronary syndrome. Coronary artery disease is one of the leading causes of mortality, morbidity worldwide. ${ }^{1}$ In developed countries, although mortality from CAD is decreasing, morbidity is increasing, particularly in older age groups. ${ }^{2}$ In developing countries, like Sri Lanka incidence of CAD is increasing with demographic changes, urbanization and lifestyle changes. ${ }^{3}$

It is clear that CAD is multi-factorial and originated as a result of both genetic and lifestyle factors. In the past, development of CAD and its association between gender, family history of coronary artery disease, diabetes mellitus, hypertension, dyslipidaemia and smoking etc. have been described and are known as established (traditional) risk factors. Integration of these factors into risk scores (the Framingham Risk Score) provides quantitative prediction of an individual's future risk of CAD. ${ }^{4}$

However, these traditional risk factors do not explain all of the risk for incident CAD events. Various new or emerging risk factors have been researched and would improve global risk assessment for CAD. Recent studies have demonstrated that additional novel markers which include elevated lipoprotein (a), homocysteine, C-reactive protein, fibrinogen, sex hormones and many others are associated with increased risk for CAD. ${ }^{5}$

Among the novel risk factors of CAD, sex hormones are known to have cardiovascular effects. Epidemiological studies have identified an inverse association between testosterone and CAD in men, ${ }^{6}$ and reduced testosterone concentration has a link to premature CAD. ${ }^{7}$ 
There are several studies that support the role of testosterone as an independent risk factor of coronary artery disease. ${ }^{6-7}$ There are studies that have shown the relationship between low endogenous testosterone levels and risk factors of CAD which include haemostatic risk factors, obesity, hypertension, dyslipidaemia, diabetes mellitus and metabolic syndrome. ${ }^{8-9}$

Among the new or emerging risk factors, particularly inflammatory markers might identify the high risk group and might benefit from more aggressive risk reduction. Atherothrombosis of the coronary vessels is considered as a disorder of inflammation and lipid accumulation. ${ }^{10}$ High-sensitivity C-reactive protein is proposed as a new coronary risk marker and may reflect either an acute or chronic inflammation. High-sensitivity C-reactive protein has attracted increasing attention in recent years, following epidemiologic studies showing hs-CRP as an independent predictor associated with risk of future cardiovascular events in those who are predisposed. ${ }^{11}$

The morbidity from non-communicable disease has increased in Sri Lanka. Therefore health and economic implications for caring these patients are enormous. Hence, identification of novel risk factors which are modifiable is of immense importance in the primary prevention. In the local setting, the association of testosterone and hs-CRP with CAD is not known, although this relationship has been well-studied in the Western population. We Sri Lankans are Asians and have different tendencies for diseases. Therefore we undertook a study to identify the relationship between low testosterone, high hs-CRP and CAD.

\section{Preliminary study}

In an attempt to find out the optimal time to obtain blood that would reflect the baseline levels of hs-CRP and testosterone in patient with acute myocardial infarction (MI), we did a preliminary study. Total testosterone and hs-CRP levels are known to fluctuate during $\mathrm{MI}$ according to the existing literature. ${ }^{12-13}$ However, the optimal time to obtain blood samples is not consistent. Since our main study evaluates the relationship between serum TT and hs-CRP concen-tration in myocardial infarction patients also, it was of paramount importance that timing of blood sampling for the basal levels of these two parameters was determined.

Therefore in eight patients admitted with acute coronary syndrome (ACS), serum TT and hs-CRP were assayed on serial blood samples (on admission, day three, day five, day 24 and day 84). We found that TT fluctuates after ACS. The optimal time to obtain blood sample that reflect the baseline TT level in a patient presenting with ACS appears to be within first three days from the onset of ACS event. If this time window is missed, blood sampling for testosterone should be delayed until 12 weeks to assess the baseline levels. ${ }^{14}$ Serum
hs-CRP fluctuates after ACS, peaked at day three, returns to the basal level by day five following ACS. Therefore basal level of hs-CRP is more reliably reflected by the on admission sample. ${ }^{15}$ Hence, we decided to collect the on admission blood sample from MI patients.

\section{Main study}

Main study included 103 consecutive male patients (age range of 30-70 years) with angiographicallydiagnosed CAD awaiting coronary artery bypass graft (CABG) at the Cardiothoracic Unit and 103 ST-elevation myocardial infarction (STEMI) patients admitted to Coronary Care Unit, Teaching Hospital, Karapitiya, Sri Lanka as cases. Male patients in the same age range, awaiting minor surgeries in surgical units and with normal electrocardiogram (ECG) and without having clinically evident CAD were selected as controls. Serum TT, hsCRP, lipids and plasma glucose were estimated. Three methods were employed in assessing the severity of atherosclerotic burden which included Gensini, Leaman and vessel score. ${ }^{16-18}$ In STEMI patients, Killip classes, TIMI (Thrombolysis In Myocardial Infarction), GRACE (Global Registry of Acute Coronary Events) and modified Selvester ECG (electrocardiographic) QRS scores were used in assessing the severity of myocardial infarction. ${ }^{19-22}$

We found that TT levels were low, while hs-CRP levels were high in patients with angiographically-proven CAD compared to controls (Table 1 ). The hs-CRP showed a negative correlation with TT (Figure 1). The low levels of $\mathrm{TT}$, high levels of hs-CRP and abnormal lipid profile appeared to be risk factors in atherogenesis. Testosterone level did not show a correlation with the severity of CAD, while hs-CRP level did show a significant positive correlation with the severity. ${ }^{23}$

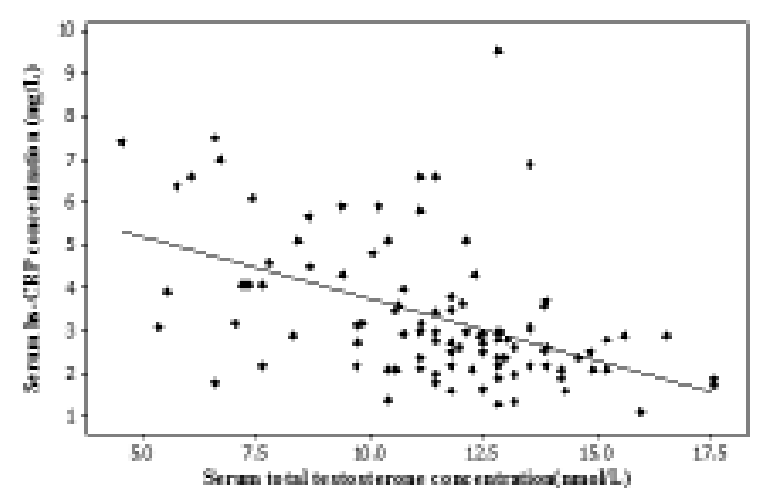

Figure 1. Negative correlation between serum total testosterone and hs-CRP concentrations.

We showed that serum hs-CRP levels were high both in patients with established CAD and ST- elevation myocardial infarction compared to controls. A significant negative correlation was observed between hs-CRP and 
Table 1. Comparison of laboratory findings between angiographically-proven CAD patients and controls

\begin{tabular}{lclc}
\hline Measurements & $\begin{array}{c}\text { Angiographically proven } \\
\text { CAD patients } \\
n=103\end{array}$ & $\begin{array}{c}\text { Controls } \\
n=103\end{array}$ & $p$ \\
\hline TT (nmol/L) & $11.4 \pm 2.7$ & $18.1 \pm 7.2$ & 0.001 \\
hs-CRP (mg/L) & $3.4 \pm 1.62$ & $1.7 \pm 0.6$ & 0.001 \\
TGs (mmol/L) & $2.5 \pm 1.0$ & $1.5 \pm 0.8$ & 0.001 \\
TCh $(\mathrm{mmol} / \mathrm{L})$ & $5.9 \pm 2.8$ & $5.2 \pm 1.6$ & 0.022 \\
HDL-Ch (mmol/L) & $1.1 \pm 0.5$ & $1.4 \pm 0.6$ & 0.001 \\
LDL-Ch (mmol/L) & $3.9 \pm 1.2$ & $3.1 \pm 0.5$ & 0.001 \\
PG (mmol/L) & $5.5 \pm 1.4$ & $5.1 \pm 0.6$ & 0.007 \\
\hline
\end{tabular}

All values expressed as mean $\pm \mathrm{SD}$. $p$-Values stated calculated by two-sample $t$-test. CAD = Coronary artery disease, TT = Total testosterone, hs-CRP = High sensitivity-C-reactive protein, TGs = Triglycerides, $\mathrm{TCh}=$ Total cholesterol, HDL-Ch = High density lipoprotein cholesterol, LDL-Ch = Low density lipoprotein cholesterol, PG = Plasma glucose .

HDL-Ch (Figure 2), while significant positive correlations were seen between hs-CRP and other lipid measurements. A significant positive correlation existed between hs-CRP and waist and hip circumferences. Therefore higher levels of hs-CRP appear to be promoting dyslipideamia associated with obesity and it is a marker of atherogenic milieu reflecting the chronic inflammatory process involved. ${ }^{24}$

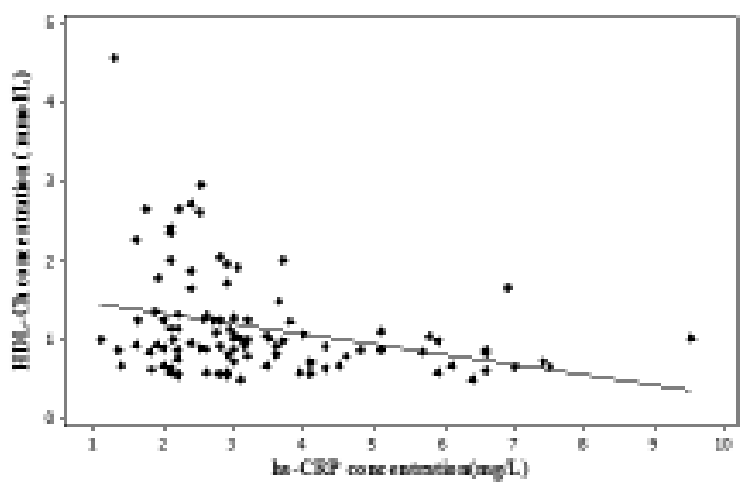

Figure 2. Negative correlation between serum hs-CRP and HDL-Ch concentrations.

Moreover, we were interested to find out the prevalence of cardiovascular risk factors (CVRFs), socioeconomic determinants and to explore their relationship between CVRFs and the angiographic severity of CAD. Therefore 141 male patients with angiographically-diagnosed CAD who were awaiting CABG at Cardiothoracic Unit, Teaching Hospital, Karapitiya were selected. We found that majority of the patients were from lower socioeconomic status and the conventional CVRFs were not able to predict the CAD severity assessed by the angiographic based scores, although they were prevalent. ${ }^{25}$
In order to clarify the relationship between serum TT and lipid parameters in angiographically confirmed CAD patients, we selected 103 angiographically proven CAD patients awaiting CABG and 103 controls. Fasting plasma glucose, serum total cholesterol (TCh), triglycerides (TGs), HDL-Ch, LDL-Ch and TT levels were estimated. The variables were compared and the relationships were assessed using correlation coefficients. There was a significant positive association between serum TT and HDL-Ch in both groups (Figure 3). Anegative association was found between testosterone and LDL-Ch in patients with CAD. Hence, low levels of testosterone appear to associate with an atherogenic lipid milieu. ${ }^{26}$

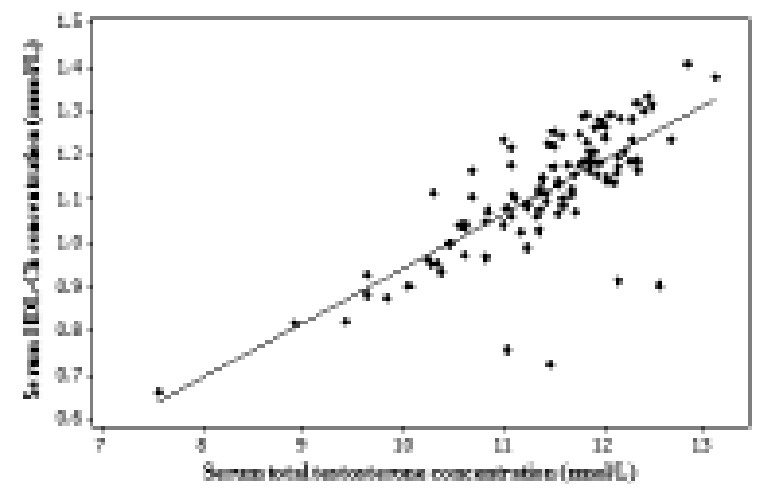

Figure 3. Positive correlation between serum total testosterone and HDL-cholesterol concentrations.

We evaluated the relationship between anthropometric measurements and serum TT level and wanted to find out the most reliable anthropometric measurement that can predict the testosterone deficiency. Obesity and anthropometric parameters are related to the testosterone concentration in men, although debate remains as to which anthropometric parameters are most 
important. Fat mass or adiposity is an important negative determinant of serum TT level. Therefore the relationship between weight, height, waist and hip circumferences, body mass index (BMI) and waist-to-hip ratio and baseline TT level was analysed in three hundred and nine male subjects. We found that there is an inverse association between anthropometric variables and serum TT level. Waist circumference was the most reliable predictor of testosterone deficiency among the anthropometric measurements. ${ }^{27}$

Premature CAD in men has been linked to low testosterone. ${ }^{7}$ Therefore we investigated the association of low TT levels with premature CAD in our patient group. Thirty one men, 45 years of age or below with documented CAD constituted the cases. Control group consisted of 31 men below the same age, without having clinically evident CAD. The serum TT was significantly lower in patients with premature CAD compared to controls. ${ }^{28}$

We further found that mean basal (on admission) serum TT in STEMI patients was significantly lower than controls (Table 2). The difference remained statistically significant after adjustment for confounding variables. Serum TT was found to be an independent predictor of both angiographically-proven CAD and STEMI patients. Low TT level showed high adjusted (age, BMI, smoking) odds ratio as a risk factor for both angiographicallyproven CAD and STEMI. The mean basal (on admission) hs-CRP concentration in STEMI patients was significantly higher compared to controls. In STEMI patients the TT level did not show a significant association with the severity of STEMI assessed by cTnI, Killip classes, TIMI, GRACE and ECG score, but it showed a significant association with left ventricular ejection fraction. However, hs-CRP level showed a significant positive association with the severity assessed by Killip classes, TIMI, GRACE and ECG score. Testosterone was independently related to the development of in-hospital complications, but not beyond this period according to our study. ${ }^{29}$

\section{Summary}

Mean basal serum total TT level was significantly lower, while baseline hs-CRP level was significantly higher in patients with angiographically-proven CAD patients and patients with ST-elevation myocardial infarction (STEMI) compared to controls. Total testosterone was an independent risk factor for CAD in both angiographically-proven CAD and STEMI patient groups. Low levels of TT showed a high odds ratio as a risk factor for CAD in both patient groups. Serum lipids and plasma glucose were significantly higher both in STEMI patients and angiographically-proven CAD patients compared to controls. A significant negative correlation was observed between hs-CRP and serum TT. Serum TT showed a significant positive correlation with HDL-cholesterol, while it showed a significant negative correlation with LDL-cholesterol. High-sensitivity-CRP showed a significant negative correlation with HDL-cholesterol, while it showed a significant positive correlation with LDLcholesterol.

Total testosterone did not show a significant correlation with the severity of CAD assessed by angiographic scores in angiographically-proven coronary artery disease patients, while hs-CRP displays a significant positive correlation. Severity of myocardial infarction graded by Killip classes, clinical risk scores (TIMI and GRACE) and ECG score was not significantly correlated with total testosterone concentration, but significantly correlated with hs-CRP concentration.

Table 2. Comparison of laboratory findings between STEMI patients and controls

\begin{tabular}{llll}
\hline Measurements & $\begin{array}{c}\text { STEMI patients } \\
n=103\end{array}$ & $\begin{array}{c}\text { Controls } \\
n=103\end{array}$ & $p$ \\
\hline TT (nmol/L) & $11.4 \pm 3.2$ & $18.1 \pm 7.2$ & 0.001 \\
hs-CRP (mg/L) & $3.7 \pm 0.84$ & $1.7 \pm 0.60$ & 0.001 \\
TGs (mmol/L) & $2.1 \pm 1.0$ & $1.5 \pm 0.8$ & 0.001 \\
TCh (mmol/L) & $6.0 \pm 2.4$ & $5.1 \pm 1.6$ & 0.001 \\
HDL-Ch (mmol/L) & $1.1 \pm 0.5$ & $1.3 \pm 0.6$ & 0.001 \\
LDL-Ch (mmol/L) & $4.5 \pm 2.4$ & $3.1 \pm 0.5$ & 0.001 \\
PG (mmol/L) & $6.0 \pm 2.06$ & $5.0 \pm 0.6$ & 0.001 \\
\hline
\end{tabular}

All values expressed as mean \pm SD. $p$-Values stated calculated by two-sample $t$-test.

TT = Total testosterone, hs-CRP = High sensitivity C-reactive protein, TGs = Triglycerides, $\mathrm{TCh}=$ Total cholesterol, $\mathrm{HDL}-\mathrm{Ch}$ = High density lipoprotein cholesterol, LDL-Ch = Low density lipoprotein cholesterol, PG = Plasma glucose 
We showed that low levels of total testosterone, high levels of hs-CRP, abnormal lipid profile and elevated plasma glucose are associated with an atherogenic milieu. Low levels of total testosterone and high level of hs-CRP can be perceived as risk factors of coronary artery disease. Majority of patients with CAD were from low socioeconomic background.

\section{Acknowledgements}

I wish to express my sincere gratitude to both of my supervisors; Prof. M. R. Mohideen, Senior Professor in Internal Medicine, International Medical University IMU Clinical School, Malaysia and Prof. Chitra Pathirana, Senior Professor of Biochemistry, Department of Biochemistry, Faculty of Medicine, University of Ruhuna, Galle, Sri Lanka for the enormous guidance and encouragement given to me to make my endeavour a success. I extend my gratitude to Dr. B. P. S. Withanawasam, Cardiologist, Teaching Hospital, Kegalle, Sri Lanka for the valuable services rendered in interpreting the coronary angiograms and formulating the severity scores. I wish to express my gratitude to Mrs. D.A.B.N.Amerasekara (Statistician, Applied Statistic Association, Sri Lanka), Senior Lecturer, Department of Crop Science, Faculty of Agriculture, University of Ruhuna, Sri Lanka for the support offered in data analysis. I wish to acknowledge the University Grants Commission, Sri Lanka for funding the project (Grant No: UGC/ICD/CRF/2009/2/47).

\section{References}

1. World Health Organization. Cardiovascular diseases: Health topics. 2012. Available at:

http://www.who.int/mediacentre/factsheets/fs317/en/ index.html.

2. Subcommittee. A report from the American Heart Association Statistics Committee and Stroke Statistics Subcommittee. Circulation 2008; 117(4): e25-146.

3. Annual Health Bulletin, Ministry of Health, Sri Lanka, 2007. Available at:

http://203.94.76.60/AHB2007/Annual Health Statistics 2007.html.

4. D'Agostino RB Sr, Grundy S, Sullivan LM, Wilson P. Validation of the Framingham coronary heart disease prediction scores: results of a multiple ethnic groups investigation. The Journal of the American Medical Association 2001; 286(2):180-87.

5. Wilson PW. Assessing coronary heart disease risk with traditional and novel risk factors. Clinical Cardiology 2004; 27(6 Suppl 3): III7-III11.

6. Hu X, Rui L, Zhu T, Xia H, Yang X, Wang X, et al. Low testosterone level in middle-aged male patients with coronary artery disease. European Journal of Internal Medicine 2011; 22(6): e133-e36.

7. Turhan S, Tulunay C, Güleç S, Ozdöl C, Kilickap M, Altin $\mathrm{T}$, et al. The association between androgen levels and premature coronary artery disease in men. Coronary Artery Disease 2007; 18(3):159-62.

8. Allan CA, McLachlan RI. Androgens and obesity. Current Opinion in Endocrinology, Diabetes, and Obesity 2010; 17(3): 224-32.

9. Vikan T, Schirmer H, Njølstad I, Svartberg J. Low testosterone and sex hormone-binding globulin levels and high estradiol levels are independent predictors of type 2 diabetes in men. European Journal of Endocrinology 2010; 162(4): 747-54.

10. Choi J, Joseph L, Pilote L. Obesity and C-reactive protein in various populations: a systematic review and metaanalysis. Obesity Reviews 2013; 14(3): 232-44.

11. Ridker PM, Rifai N, Rose L, Buring JE, Cook NR. Comparison of $\mathrm{C}$-reactive protein and low density lipoprotein cholesterol levels in the prediction of first cardiovascular events. The New England Journal of Medicine 2002; 347(20): 1557-65.

12. Cusack MR, Marber MS, Lambiase PD, Bucknall CA, Redwood SR. Systemic inflammation in unstable angina is the result of myocardial necrosis. Journal of the American College of Cardiology 2002; 39(12): 1917-23.

13. Pugh PJ, Channer KS, Parry H, Downes T, Jone TH. Bioavailable testosterone levels fall acutely following myocardial infarction in men: association with fibrinolytic factors. Endocrine Research 2002; 28(3): 161-73.

14. Wickramatilake CM, Mohideen MR, Pathirana C. Fluctuations of testosterone in acute coronary syndrome. Sri Lanka Journal of Diabetes Endocrinology and Metabolism 2013; 3: 8-11.

15. Wickramatilake CM, Mohideen MR, Pathirana C. Fluctuations of high sensitivity C-reactive protein in acute coronary syndrome. Galle Medical Journal 2014; 19(1): 11-15.

16. Gensini GG. A more meaningful scoring system for determining the severity of coronary heart disease. The American Journal of Cardiology 1983; 51(3): 606.

17. Leaman DM, Brower RW, Meester GT, Serruys P, van den Brand M. Coronary artery atherosclerosis: Severity of the disease, severity of angina pectoris and compromised left ventricular function. Circulation 1981; 63(2): 285-99.

18. Ringqvist I, Fisher LD, Mock M, Davis KB, Wedel H, Chaitman BR, et al. Prognostic value of angiographic indices of coronary artery disease from the Coronary Artery Surgery Study (CASS). The Journal of Clinical Investigations 1983; 71(6): 1854-66.

19. Killip T. 3rd, Kimball JT. Treatment of myocardial infarction in a coronary care unit. A two year experience with 250 patients. The American Journal of Cardiology 1967; 20(4): 457-64.

20. Morrow DA, Antman EM, Charlesworth A, Cairns R, Murphy SA, de Lemos JA, et al. TIMI risk score for ST-elevation myocardial infarction: a convenient, bedside, clinical score for risk assessment at presentation: an intravenous nPA for treatment of infarcting myocardium early II trial substudy. Circulation 2000; 102(17): 2031-37. 
21. Eagle KA, Lim MJ, Dabbous OH, Pieper KS, Goldberg $\mathrm{RJ}$, Van deWerf $F$, et al. A validated prediction model for all forms of acute coronary syndrome: estimating the risk of 6-month postdischarge death in an international registry. The Journal of the American Medical Association 2004; 291(22): 2727-33.

22. Wagner GS, Freye CJ, Palmeri ST, Roark SF, Stack NC, Ideker RE, et al. Evaluation of a new QRS scoring system for estimating myocardial infarct size. I. Specificity and observer agreement. Circulation 1982; 65(2): 42-47.

23. Wickramatilake CM, Mohideen MR, Withanawasam BPS, Pathirana C. Testosterone, high sensitive C-reactive protein in coronary artery disease patients awaiting coronary artery bypass graft. Andrologia 2015; 47(5): 493-98.

24. Wickramatilake CM, Mohideen MR, Pathirana C. Association of serum testosterone with the complications of acute myocardial infarctions. Pakistan Heart Journal 2015; 48(01): 22-9.

25. Wickramatilake CM, Withanawasam BPS, Mohideen MR. Cardiovascular risk factors, socioeconomic determinants and angiographic severity of coronary artery disease in patients awaiting coronary artery bypass graft in a provincial hospital Sri Lanka. Galle Medical Journal 2014; 19(2): 12-17.

26. Wickramatilake CM, Mohideen MR, Pathirana C. Highsensitivity C-reactive protein in Sri Lankan males with coronary artery disease. Bangladesh Journal of Medical Sciences 2016; 15(1): 44-50.

27. Wickramatilake CM, Mohideen MR, Pathirana C. Association of serum testosterone with lipid abnormalities in patients with angiographically proven coronary artery disease. Indian Journal of Endocrinology and Metabolism 2013; 17(6): 1061-65.

28. Wickramatilake CM, Mohideen MR, Pathirana C. Association between anthropometric parameters and testosterone deficiency in men. Sri Lanka Journal of Diabetes, Endocrinology and Metabolism 2014; 4: 7-11.

29. Wickramatilake CM, Mohideen MR, Pathirana C. Premature coronary artery disease and testosterone in Sri Lankan men. Sri Lanka Journal of Diabetes Endocrinology and Metabolism 2014; 4: 17-21. 